\title{
Numerical Simulation of Powder Ceramics with SiC Susceptor on Hybrid Microwave Sintering
}

\author{
Mengyou Xie, Jianjun Shi* and Guoping Chen \\ School of Civil Engineering and Architecture, Southwest University of Science and Technology, Mianyang 621010, China \\ ${ }^{*}$ Corresponding author
}

\begin{abstract}
Under high-temperature conditions, the microwave sintering process is exceedingly sensitive in terms of material properties. Accordingly, the accurate measurement of temperature is infeasible when a microwave oven is utilized as the main apparatus. Therefore, a three-dimensional model built through the finite element software COMSOL Multiphysics was used to investigate the hybrid microwave sintering (HWMS) process. Numerical simulation of the entire microwave sintering process has been realized and it could reflect the actual sintering process. In this paper, the proposed numerical simulation entails four kinds of thickness of SiC rings. Specifically, three principal aspects were studied: the distribution of electric field, temperature variation and densification of powder injection molding (PIM) material. As a result, a significant "room-temperature" coupling was observed under the effect of susceptor in low dielectric-loss materials; The influence of susceptor ring thickness on temperature variation and densification of ceramic material was secured; The ultimate temperature remains steady at a certain heating time regardless of different thickness; The densification process was significant enhancement in the sintering stage.
\end{abstract}

Keywords-microwave process; sintering; simulation; $\mathrm{ZrO}_{2}$; single mode

\section{INTRODUCTION}

The merits of microwave sintering over conventional sintering process have been validated in high-temperature processing [1][2]. Specifically, the implementation of sintering densification and temperature evolution is determined by material dielectric loss. Low dielectric materials, such as $\mathrm{Al}_{2} \mathrm{O}_{3}$ and $\mathrm{ZrO}_{2}$ [3], are hard to achieve "room-temperature coupling" effect, thus there is a long warming-up time in the process of direct microwave sintering [4]. Moreover, microwave ignition included in this period leads to a disturbance of the whole sintering process. Meanwhile, a satisfied coupled effect with microwave is witnessed because of the high-loss factor of $\mathrm{SiC}$ [5]. Likewise, it can be used to solve the problem of "roomtemperature coupling" by thermal radiation to warm up the low dielectric-loss materials. Extensive efforts have been devoted to deal with this problem [6][7].

As a multi-physics coupling process, it is difficult to overview of the microwave sintering behavior of materials because of the diversity in experimental procedures. In other words, the accurate temperature measurement in microwave sintering was infeasible since common tools such as thermocouple or pyrometer provide insufficient measurement data and accuracy [8]. Meanwhile, the electromagnetic evolution during heating cannot be physically measured in spite of results are important to investigate the characteristic of microwave heating. Furthermore, it is difficult to predict the internal thermal field of the sintered material. Therefore, numerical simulation can be an effective method to estimate the response of each parameter during sintering. As a powerful software package, COMSOL is feasible to model the coupling behavior of multi-physics, including electromagnetic, heat transfer and the densification process of powder material [9]. The microwave dissipated power calculated from the electromagnetic equation provides a heating source that forms the inhomogeneous term of the heat transport equation.

The sintering process is determined by material properties such as thermal conductivity, specific heat capacity, complex permittivity and magnetic permeability, especially. All these parameters are temperature-dependent under high-temperature conditions. Although there were some studies related to the simulation of microwave sintering process [10][11], the effect of temperature-dependent materials paraments has not been considered. The main objective of present paper is to suppose a finite element method to simulate the Multiphysics phenomena, coupling electromagnetic, heat transfer and mechanics model, with main consideration of the heat flux and heat radiation effects as well as the influence of temperature-dependence parameters. Three principal aspects were mainly investigated: (1) the distribution of electric field in the cavity; (2) the temperature evolution of PIM zirconia material; (3) the densification process of PIM zirconia material. The assist heating effects of $\mathrm{SiC}$ susceptor were also incorporated in the model.

\section{MATERIALS AND GEOMETRIC MODEL}

\section{A. Geometry Model}

The numerical simulation was performed in sing-mode microwave cavity with vacuum condition, which was connected to a $2.45 \mathrm{GHz}$ microwave source via a rectangular waveguide operating in the $\mathrm{TE}_{105}$ mode. The resonant cavity has a rectangular cross section and microwave produced by a magnetron were transported along the $y$ axis in a rectangular waveguide $(a \times b=43.18 \mathrm{~mm} \times 86.36 \mathrm{~mm})$ of the same cross section and transferred to the cavity through an iris, the input power was $1000 \mathrm{~W}$.

Since it is easily lead to cracking by fast sintering, the sample was not located in places with high electric field intensity in present work. SiC susceptor was placed around the sample for assist heating, with different thickness ( $d$ varies from 1 4 mm) and inside diameter remains $6 \mathrm{~mm}$. Notably, the susceptor did not completely shield the electric field. The thermal insulation cylinder was set to reduce heat loss, the insulation cylinder loss factor considered 0 , which gave little effect to the electric field distribution in present study. The three-dimensional geometric model was developed in Figure I. 


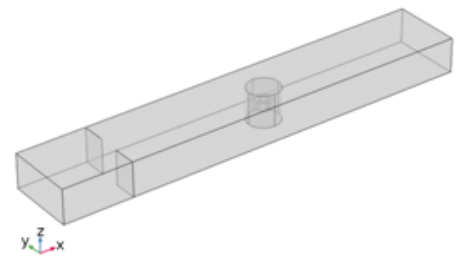

(A)

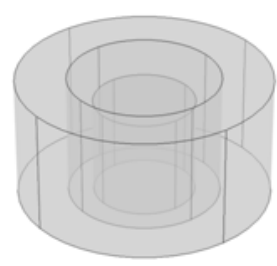

(B)
FIGURE I. THE GEOMETRIC MODEL: (A) CAVITY (B) SILICON CARBIDE SUSCEPTOR RING AROUND ZIRCONIA SAMPLE

\section{B. Material Properties}

PIM zirconia ceramic was in fact a porous medium. Its porosity decreases during sintering process. Assuming the injected samples for sintering are formed with continuous phase, the effective dielectric properties of the porous medium at a given porosity are calculated according to the Maxwell-Wagner mixture rule:

$$
\varepsilon_{\mathrm{eff}}^{*}=\frac{\varepsilon_{s}^{*}\left[\varepsilon_{a}^{*}(1+2 \Theta)+2 \varepsilon_{s}^{*}(1-\Theta)\right]}{\varepsilon_{a}^{*}(1-\Theta)+\varepsilon_{s}^{*}(2+\Theta)}
$$

where $\varepsilon_{a}^{*}$ and $\varepsilon_{S}^{*}$ is the relative complex dielectric constants of the air and $\mathrm{ZrO}_{2}$, respectively. $\Theta$ is the porosity. The heat capacity of per unit volume $\left(\rho C_{p}\right)_{\text {eff }}$ of porous zirconia is:

$$
\left(\rho C_{p}\right)_{\text {eff }}=(1-\Theta)\left(\rho C_{p}\right)_{s}+\Theta\left(\rho C_{p}\right)_{a}
$$

where $\left(\rho C_{p}\right)_{s},\left(\rho C_{p}\right)_{a}$ are the values for zirconia and air, respectively. The thermal conductivity $\kappa_{\text {eff }}$ of porous zirconia is:

$$
2 \kappa_{\text {eff }}^{2}+\left[\kappa_{s}(3 \Theta-2)+\kappa_{a}(1-3 \Theta)\right] \kappa_{\text {eff }}-\kappa_{s} \kappa_{a}=0
$$

where $\kappa_{s}, \kappa_{a}$ are the thermal conductivity $(\mathrm{W} /(\mathrm{m} \cdot \mathrm{K}))$ of full dense zirconia and air, respectively [12-16].

\section{MATHEMATICAL METHOD}

\section{A. Governing Equations}

Electromagnetic waves solved in a frequency domain and the transient heat transfer physics are coupled to each other during the whole sintering process. In each time step, the calculation of the electromagnetic field and heat transfer must be updated. Maxwell's equation is used to obtain the electromagnetic field:

$$
\nabla \times \mu_{r}^{-1}(\nabla \times \mathbf{E})-k_{0}^{2}\left(\varepsilon_{r}^{\prime}-\frac{i \sigma}{\omega \varepsilon_{0}}\right) \mathbf{E}=0
$$

where $\mu_{r}$ is the relative permeability, $\mathbf{E}$ is the electric field $(\mathrm{V} / \mathrm{m})$ $\varepsilon_{0}$ is the vacuum dielectric constant, $\varepsilon^{\prime}$ is the relative dielectric constant, $\sigma$ is the electric conductivity $(\mathrm{S} / \mathrm{m}), \omega$ is the angular frequency $(\mathrm{rad} / \mathrm{s})$. The heat conduction equation is given as:

$$
\left(C_{p} \rho\right)_{\mathrm{eff}} \frac{\partial T}{\partial t}+\nabla \cdot\left(\left(C_{p} \rho\right)_{\mathrm{eff}} \mathbf{u} \cdot T-\kappa_{\mathrm{eff}} \nabla T+q_{r}\right)=Q_{e m}
$$

where $T$ is the temperature $(\mathrm{K}), q_{r}$ is the heat flux $(\mathrm{W} / \mathrm{m}), Q_{e m}$ is a volumetric source term due to microwave power absorption $\left(\mathrm{W} / \mathrm{m}^{3}\right)$. When the electromagnetic wave interacts with dielectric materials, part of the electromagnetic energy will be converted into heat:

$$
Q_{e m}=Q_{r h}+Q_{m l}
$$

where the resistive loss is:

$$
Q_{r h}=\frac{1}{2} \operatorname{Re}\left(\mathbf{J} \cdot \mathbf{E}^{*}\right)
$$

And the magnetic loss is:

$$
Q_{m l}=\frac{1}{2} \operatorname{Re}\left(i \omega \mathbf{B} \cdot \mathbf{H}^{*}\right)
$$

where $\mathbf{J}$ is the current density $\left(\mathrm{A} / \mathrm{m}^{2}\right), \mathbf{B}$ is the magnetic flux density $\left(\mathrm{Wb} / \mathrm{m}^{2}\right)$, and $\mathbf{H}$ is the magnetic field intensity $\left(\mathrm{A} / \mathrm{m}^{2}\right)$.

\section{B. Densification Law}

The densification process is related to the sintering stress and the microwave promotive effect [17]. Densification is a thermally activated process. The activation energy for sintering is the key factor to determine the densification rate, as shown in (9) [18].

$$
\frac{d \rho_{r}}{d t}=C \exp \left(-E_{a} / R T\right)
$$

The existing densification constitutive for PIM Zirconia ceramics has been introduced into the present work, as shown in (10) [10]:

$$
\frac{d \rho_{r}}{d t} \frac{1}{\rho_{r}}=200 e^{(-20000 / T)}\left(\frac{1-\rho_{r}}{\rho_{r}-\rho_{0}}\right)^{2}
$$

where $\rho_{0}$ is the initial relative density, taking value of 0.65 in present simulation.

\section{Boundary Condition}

The impedance boundary condition is imposed on the metal wall of the microwave cavity, where the electromagnetic field is regarded to penetrate only a short distance outside the boundary, given as:

$$
\sqrt{\frac{\mu_{0} \mu_{r}}{\varepsilon_{0} \varepsilon_{r}-i \sigma / \omega}} \mathbf{n} \times \mathbf{H}+\mathbf{E}-(\mathbf{n} \cdot \mathbf{E}) \mathbf{n}=\left(\mathbf{n} \cdot \mathbf{E}_{s}\right) \mathbf{n}-\mathbf{E}_{s}
$$

The rectangular port was excited by a transverse electric wave. The boundary condition consists a parameter of 
propagation constant $\beta$, which is given by the expression as:

$$
\beta=\frac{2 \pi}{c} \sqrt{(v)^{2}+\left(v_{c}\right)^{2}}
$$

where $v$ is the frequency $(\mathrm{Hz}), v_{c}$ is a cutoff frequency $(\mathrm{Hz})$. When the rectangular port is excited, the heat convection of sample surface and surrounding air is defined as:

$$
-\mathbf{n} \cdot \mathbf{q}_{\text {conv }}=h \cdot\left(T_{\text {ext }}-T\right)
$$

where $\mathbf{q}_{c o n v}$ is the convection heat flux $(\mathrm{W} / \mathrm{m}), h$ is heat transfer coefficient $(\mathrm{W} /(\mathrm{m} \cdot \mathrm{K})), T_{e x t}$ is the room temperature. Heat radiation of the susceptor to the sample is:

$$
-\mathbf{n} \cdot \mathbf{q}_{\text {rad }}=\varepsilon \sigma \cdot\left(T_{\text {ext }}^{4}-T^{4}\right)
$$

where $\mathbf{q}_{\mathrm{rad}}$ is the thermal radiative heat flux $(\mathrm{W} / \mathrm{m}), \varepsilon$ is the surface emissivity.

\section{RESULT}

\section{A. Distribution of Electric Field and Temperature Field}

The electric field propagates through the iris in the cavity. Insulating material has little effect on the electric field. An ambient temperature of $300 \mathrm{~K}$ was assumed for the simulation. Figure II gives the results of electric field in cases with susceptor ring thickness is $4 \mathrm{~mm}$ at initial sintering. Due to the dielectric loss of $\mathrm{SiC}$ (27.99) is much greater than $\mathrm{ZrO}_{2}(0.1 \sim 1)$, it absorbs most of the electric field and is heated up more quickly, then leads to higher final temperature.

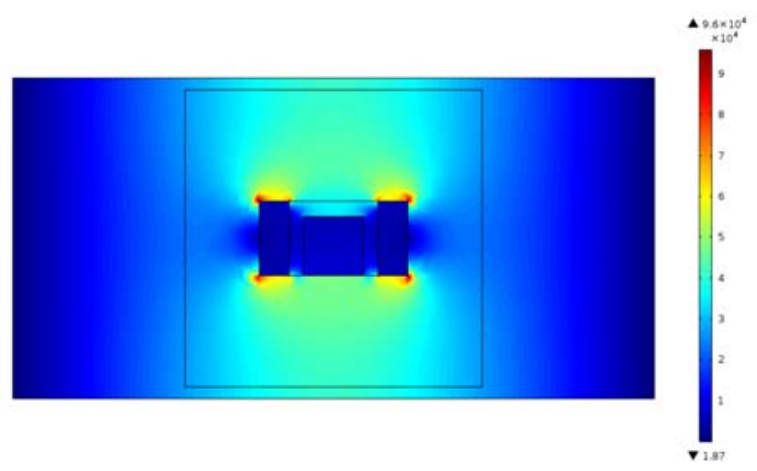

FIGURE II. THE DISTRIBUTION OF ELECTRIC FIELD

The absorption power for zirconia along $z$-axis is shown in Figure III. It details the absorbed microwave power within the zirconia ceramic at the initial sintering stage. The intense radiant heat caused by $\mathrm{SiC}$ lead the absorbed power sudden change suddenly at the zirconia sample surface. The power absorbed in zirconia ceramic made it virtually no attenuation in the sample, owing to the high penetration depth and standing wave patterns give rise to a power peak in the center of the sample.

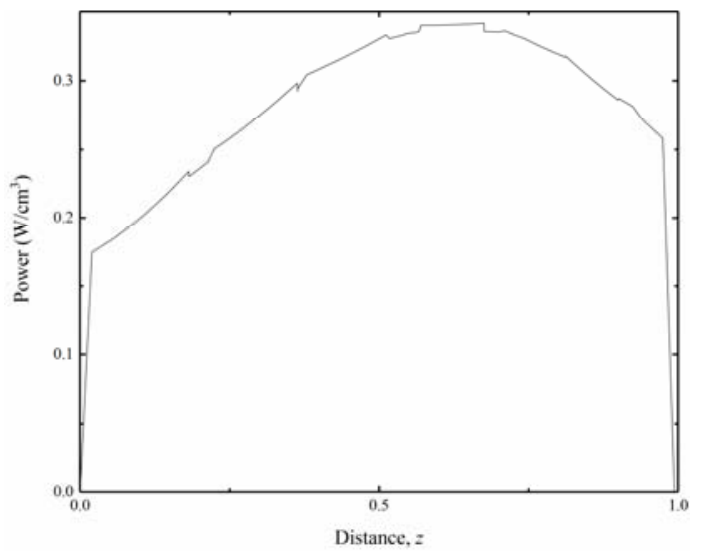

FIGURE III. ABSORPTION POWER INSIDE ZIRCONIA SAMPLE

Figure IV provides the the evolution of temperature field, the maximum temperature $T_{\max }$ and the difference between maximum temperature and minimum temperature $\left(\Delta T=T_{\max }{ }^{-}\right.$ $\left.T_{\min }\right)$ within the sample are chosen to be calibration parameter. It shows that the sample was rapidly heated at a rate of about $300 \mathrm{~K} \cdot \mathrm{min}^{-1}$ at the first $250 \mathrm{~s}$ through heat radiation from $\mathrm{SiC}$ susceptor ring. It indicates that the susceptor can assist the sintered compacts to reach the dielectric-coupling temperature in a short time. Then the heating rate is gradually slowed down because there is a certain heat loss and the low thermal conductivity within the zirconia. As the thickness increasing, the $\Delta T$ gradually decreases; the ultimate $T_{\max }$ remained steady with a small difference.

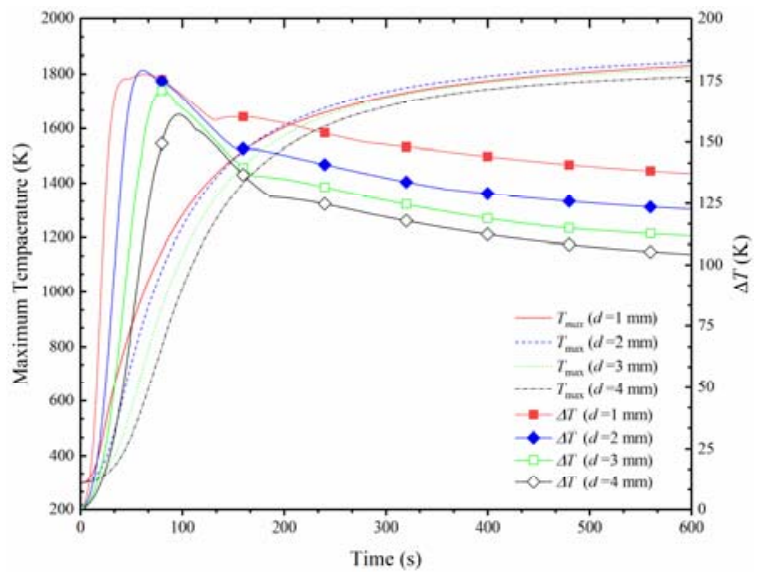

FIGURE IV. EVOLUTION OF TEMPERATURE WITH DIFFERENT SUSCEPTOR THICKNESS

\section{B. Density Evolution}

The densification process of porous compacts happens in the high temperature stage at $T=800 \mathrm{~K}$ and enhances significantly at $T=1000 \mathrm{~K}$. At a certain sintering time, the final relative density of the sintered compacts reaches more than $90 \%$ in all cases with different susceptor thickness. The peak value reaches $94.8 \%$ in the case with the thickness $1 \mathrm{~mm}$. Figure $\mathrm{V}$ gives the results of the evolution of relative density in cases with different $\mathrm{SiC}$ susceptor thickness. 


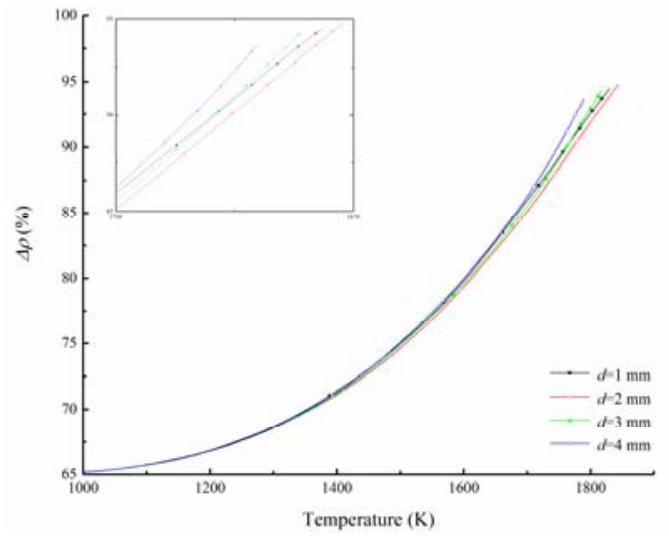

FIGURE V. THE RELATIVE DENSITY EVOLUTION IN CASES WITH DIFFERENT SUSCEPTOR THICKNESS

Figure VI plots the $\Delta T$ and the difference of relative density $\left(\Delta \rho=\rho_{r, \max }-\rho_{r, \min }\right)$ in cases with different susceptor thickness. $\Delta T$ varies from $137.4 \mathrm{~K}$ to $104.3 \mathrm{~K}$ while susceptor thickness varies from $1 \mathrm{~mm}$ to $4 \mathrm{~mm}$. The thicker susceptor ring showed a more uniform distribution of temperature, also it led a more uniform densification. $\Delta \rho$ varied from $3.80 \%$ to $3.13 \%$ with susceptor thickness varied from $1 \mathrm{~mm}$ to $4 \mathrm{~mm}$. Because the electric field within the sintered compacts will decrease while the susceptor thickness increases. The low electric field strength results in the uneven distribution of electric field, but it is not significant. In most cases overheating is induced by obviously nonuniform distribution of the electric field inside the product [19], HMWS inhibits the risk of thermal runaway phenomenon.

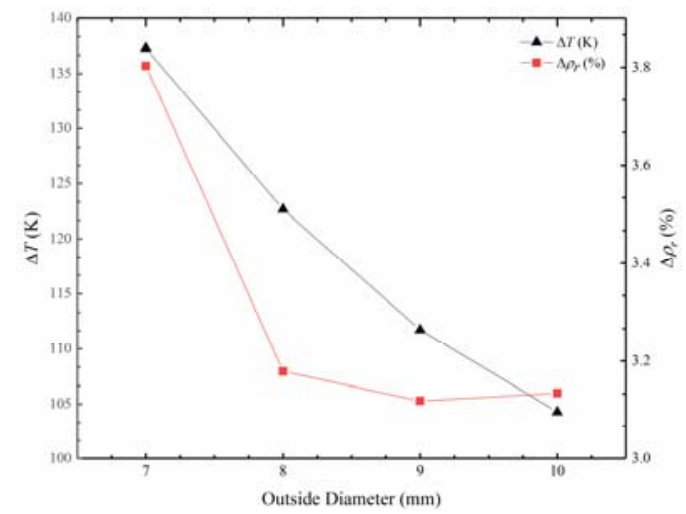

FIGURE VI. THE DIFFERENCE OF FINAL TEMPERATURE AND RELATIVE DENSITY IN CASES WITH DIFFERENT SUSCEPTOR THICKNESS

\section{CONCLUSION}

Numerical simulation of the entire microwave sintering process has been realized in the present paper. First, the distribution of electric field in the single-mode cavity has been showed, containing a cylindrical zirconia sample and $\mathrm{SiC}$ susceptor ring. Then the evolution of temperature and densification within the sintered compacts in cases with different susceptor thickness are investigated. It is notable that HMWS can also lead to a fast sintering, because the significant auxiliary heating effect happens when processing these lowdielectric materials. The significant densification process happens particularly in the sintering stage; the ultimate temperature remains steady at a certain heating time and will not be influenced by the different susceptor thickness. In present work, thermal radiation effect has been taking into account from the susceptor to the compact, and other heating losses have also been considered. However, for more accurate simulations, accurate parameters and constitutive laws for PIM zirconia material are required. It should be achieved reliably by experimental means in future work.

\section{REFERENCES}

[1] Clark D E, Sutton W H, "Microwave Processing of Materials.” Materials research society., 1996.

[2] Bykov Y V, Rybakov K I, Semenov K E. "High-temperature microwave processing of materials.” J. Phys. D Appl Phys 34.13(2001): R55.

[3] Xie Z, Yang J, Huang X, Huang Y, "Microwave processing and properties of ceramics with different dielectric loss." J. Eur. Ceram Soc., 1999, 19(3): 381-387.

[4] Janney M A, Calhoun C L, Kimrey H D, "Microwave Sintering of Solid Oxide Fuel Cell Materials: I, Zirconia - 8mol\% Yttria.” J. Am. Ceram. Soc., 1992, 75(2): 341-346.

[5] Heuguet R, Marinel S, Thuault A, Badev A, "Effects of the Susceptor Dielectric Properties on the Microwave Sintering of Alumina." J. Am. Ceram. Soc., 2013, 96(12): 3728-3736.

[6] Zhao C, Vleugels J, Groffils C, Luypaert P J, Biest O V D, "Hybrid sintering with a tubular susceptor in a cylindrical single-mode microwave furnace." Acta Mater., 2000, 48(14): 3795-3801.

[7] Charmond S, Carry C P, Bouvard D, "Densification and microstructure evolution of Y-Tetragonal Zirconia Polycrystal powder during direct and hybrid microwave sintering in a single-mode cavity." J. Eur. Ceram Soc., 2010, 30(6): 1211-1221.

[8] Croquesel J, Bouvard D, Chaix J M, Carry C P, Saunier S, "Development of an instrumented and automated single mode cavity for ceramic microwave sintering: Application to an alpha pure alumina powder." Mater. Des., 2015, 88: 98-105.

[9] Kumar C, Joardder M U H, Farrell T W, Karim M A, "Multiphase porous media model for intermittent microwave convective drying (IMCD) of food.” Int. J. Therm. Sci., 2016, 104: 304-314.

[10] Shukla A K, Mondal A, Upadhyaya A, "Numerical modeling of microwave heating." Science of Sintering, 2010, 42(1): 99-124.

[11] Law M C, Liew E L, Chang S L, Chan Y S, Leo C P, "Modelling microwave heating of discrete samples of oil palm kernels." Appl. Therm. Eng., 2016, 98: 702-726.

[12] Bouvard D, Charmond S, Carry C P, "Multiphysics Simulation of Microwave Sintering in a Monomode Cavity." 2010.

[13] Swain M V, Johnson L F, Syed R, Hasselman D P H, "Thermal diffusivity, heat capacity and thermal conductivity of porous partially stabilized zirconia.” J. Mater. Sci. Lett., 1986, 5(8): 799-802.

[14] Limarga A M, Clarke D R, "The grain size and temperature dependence of the thermal conductivity of polycrystalline, tetragonal yttria-stabilized zirconia.” Appl. Phys. Lett., 2011, 98(21): 7421.

[15] Auerkari P, "Mechanical and Physical Properties of Engineering Alumina Ceramics." Transactions of the ASAE 1980 Vol. 23 No. 4 pp. 816-821, 1996, 23(4): 816-821.

[16] Munro R G, “Material Properties of a Sintered $\alpha$-SiC.” J. Phy. Chem. Ref. Data., 1997, 26(5): 1195-1200.

[17] Rybakov K I, Olevsky E A, Semenov V E, "The microwave ponderomotive effect on ceramic sintering." Scripta Mater., 2012, 66(12): 1049-1052.

[18] Brosnan K H, Messing G L, Agrawal D K, "Microwave Sintering of Alumina at 2.45 GHz.” J. Am. Ceram. Soc., 2010, 86(8): 1307-1312.

[19] Roussy G, Bennani A, Thiebaut J M, "Temperature runaway of microwave irradiated materials.” J. Appl. Phy., 1987, 62(4): 1167-1170. 